\title{
Performance Evaluation of Physical Layer of Mobile WiMAX System by Implementing Hybrid Channel Coding Scheme
}

\author{
Jagdish D. kene \\ Research Scholar \\ ECE Department \\ V.N.I.T. Nagpur.
}

\author{
Kishor D. Kulat \\ Professor \\ ECE Department \\ V.N.I.T. Nagpur
}

\begin{abstract}
The demand of wireless communication is high data rate along with error free communication. An error in communication can be reduced by employing proper channel coding techniques. In this paper, authors have proposed the hybrid channel coding scheme in which convolution codes and turbo codes are combined to perform together. This scheme helps to provide smooth communication with high data rate even under different channel conditions. The SNR is the threshold parameter for adaptive encoding which can be measured by the channel estimation techniques for mobile WiMAX system. Authors have also taken efforts for adding adaptive code rate and modulation schemes for optimizing the mobile WiMAX system. Use of proper adaptive coding for optimizing the performance of mobile WiMAX system can be achieved. For this newly introduced hybrid scheme, the analysis and simulation are carried out with respective to standard AWGN channel which shows improved Bit Error Rate performance and also overcomes the drawbacks such as high complexity, decoding delay and power requirement.
\end{abstract}

\section{Keywords}

Mobile WiMAX, FEC codes, Convolution codes, Turbo codes, Channel estimation.

\section{INTRODUCTION}

Mobile WiMAX is the promising technology to provide the high data transmission rate over longer distance for different types of wireless communication such as data, voice and video [1]. Mobile WiMAX also provides great deal of flexibility, scalability and good quality of services [2]. Orthogonal frequency division multiplexing has been implemented to achieve high bit rate of transmission in multipath fading environment. The OFDM causes Inter Symbol Interference (ISI) which results in the system performance degradation [4]. To minimize the system error probability, forward error correction coding has been implemented. The performance of Mobile WiMAX system using convolution codes with various code rate encoders and modulation techniques approaches the Shannon's limit. This is true for lower degree of modulation. But high data rate demands higher degrees of modulation and large constrain length, that demands more power to transmit [5]. This drawback can be overcome by introducing Turbo codes in mobile WiMAX system. Turbo codes provide virtually error free communication with lower level of modulation and power requirement [6]. Turbo codes also provide good quality of service at low signal to noise ratio (SNR) values. The operation of turbo decoder is based on iterative decoding and uses Maximum A Priority (MAP) algorithm to generate the soft output. MAP decoding algorithm uses relatively large number of iterations to achieve desirable BER performance at lower SNR. This leads to excessive time delay and computational complexity which results in increasing system complexity [11], [12].

The literature survey shows that, the convolution codes outperform for higher signal to noise ratio for useful transmission rate and quality of service, whereas turbo codes perform effectively over lower value of SNR (under worst channel conditions). Owing to these results, a new scheme has been implemented called Hybrid channel coding which includes the advantages of both convolution as well as turbo coding scheme for mobile WiMAX system.

The system parameters such as channel conditions, useful bit rate and quality of service, the adaptive channel coding technique is used for mobile WiMAX system. Depending to the feedback of channel estimation or value of SNR, system can adapt either convolution code or turbo codes. The lower modulation techniques and low code rate will be equipped for maintaining the quality of service in case of worst channel conditions, while for good channel conditions high code rate and modulation could improve useful bit rate.

The work has been carried out for providing adaptive channel coding for mobile WiMAX system. The code rate and modulation scheme are all adaptable at the same time in terms of SNR of the channel. For assuring the good quality of service with low complexity, minimum time delay and minimum power requirement, a target SNR has been fixed as the threshold. Based on this target SNR, the system can adapt optimal coding technique, code rate and modulation to seek maximum useful bit rate (throughput). The BER performance has been evaluated for the proposed system model by adapting various coding, modulation and code rate against standard AWGN channel.

The performance analysis of new scheme has been carried out through the MATLAB simulation by selecting the respective superiorities of individual coding scheme. The analysis has been made that the turbo code operates better in low SNR channel condition while convolution code performs at higher SNR.

The rest of the paper is organized as follows: Section II deals with system model for WiMAX system. The discussion about utility of convolution codes and turbo codes is made in section III. Section IV describes Simulation and result oriented on the system model. The conclusions are drawn in section V. 


\section{SYSTEM MODEL}

The block schematic representation of Mobile WiMAX system is shown in fig 1 . In the transmitter section incoming information bits are encoded with either convolution or turbo encoder according to choice of code rate. The selection of particular encoder is based on value of effective SNR of the AWGN channel. The effective SNR is obtained by employing channel estimation techniques over AWGN channel which is feedback to the SNR threshold selector. Coded data is then modulated with QPSK. The coded data is converted into a frames using serial to parallel convertor. OFDM symbol is modulated by implementing Inverse Fast Fourier transform (IFFT). The guard interval is inserted to eliminate the inter symbol interference. Finally data symbols are transmitted serially through the AWGN channel.

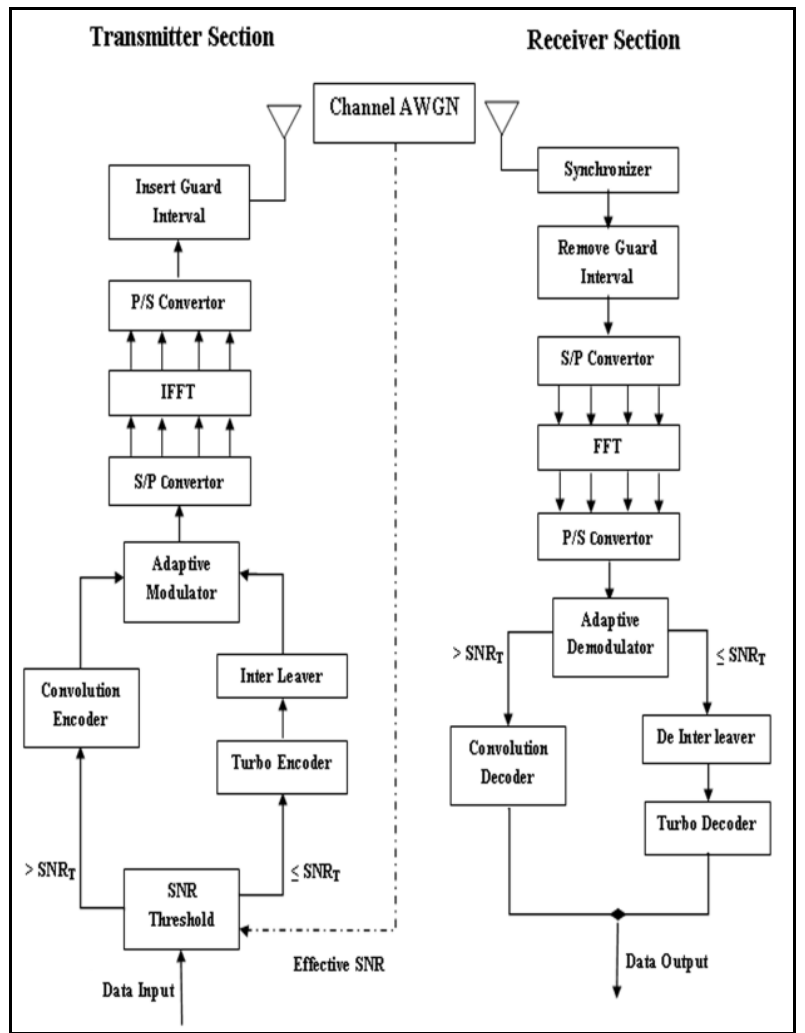

Fig 1: Block schematic of proposed Mobile WiMAX system.

At the receiver section, synchronization should be done to obtain the good strength of symbols. Guard interval ought to be removed from the received symbols. After serial to parallel conversion, the OFDM symbols are demodulated by implementing Fast Fourier Transform (FFT). Data symbols are converted into a bit sequence which is then demodulated with QPSK. Then distorted bits are decoded by either convolution or turbo decoder choosing according to the value of effective SNR obtained through channel estimation for specific data bits.

\section{CODING SCHEMES}

Forward error correction coding techniques are used to minimize the error probability of the received information. In this paper, we have discussed briefly about convolution coding and turbo coding. Authors have suggested new coding technique called Hybrid channel coding is brief in this section.

\subsection{Convolution codes}

Convolution codes are used for applications to provide good performance with low implementation cost. Convolution codes have memory that uses previous bits to encode or decode following bits. These codes are implemented by employing a binary encoder that takes a stream of information bits and converts it into a stream of transmitted bits(a codeword) [2]. Encoder consists of a shift register bank that generates the transmitted codeword according to the generator polynomials. The structure of convolution codes is shown in fig 2 with generator polynomials $(7,5)$.

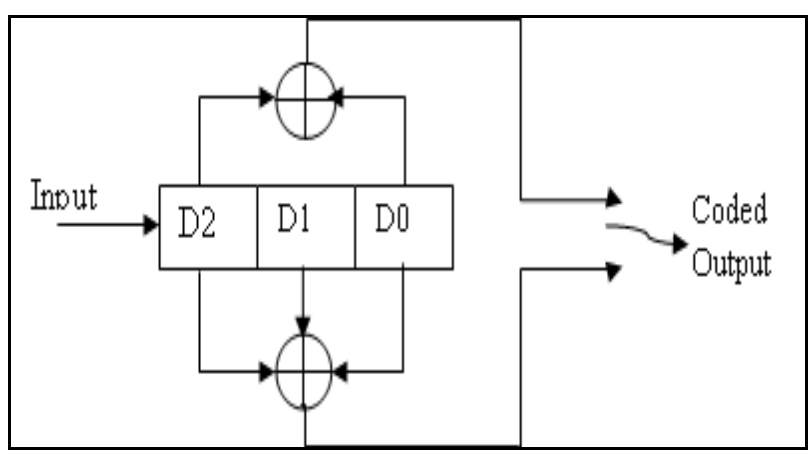

Fig 2: Convolution encoder structure for rate $1 / 2$.

To recover the information bits from channel errors, the redundancy is provided by transmitting more bits per unit time than the number of information bits per unit time. In convolution decoder, Maximum likelihood decoding can be implemented using the Viterbi algorithm. Other decoding algorithms such as SOVA (Soft Output Viterbi Algorithm) and the BCJR algorithm are also commonly used. Maximum likelihood decoding means finding the code branch in the code trellis that is most likely to transmit. Therefore, ML decoding is based on calculating the hamming distances for each branch to recover the transmitted codeword. ML algorithm is too complex, but viterbi algorithm eliminates least likely trellis path at each transmission stage and reduces decoding complexity with early rejection of unlike paths and concentrating on Survival paths of the trellis. In this way, viterbi decoding algorithm improves the efficiency of convolution codes [17].

\subsection{Turbo codes}

Turbo codes are more popular in most of the wireless applications like WiMAX, LTE etc, because of its greater Error control ability. These codes provide good BER performance and approach towards the Shannon's channel capacity limit. Turbo code provides high data rates for low order modulation schemes such as BPSK or QPSK. Turbo code is implemented by employing (i) Parallel concatenated encoder structure that uses two convolution encoders and an inter leaver to produce coded data at low power level, and (ii) Soft input soft output iterative decoders [2]. The operation of the turbo decoder is based on iterative decoding which is considered as the main feature of turbo codes. The structure of turbo decoder is shown in fig 3 [3]. Two basic iterative decoding algorithms, namely Soft Output Viterbi Algorithm (SOVA) and Maximum A Posteriori Probability (MAP) algorithm demand complex decoding operations over several iteration cycles. Among these algorithms, SOVA has the least computational complexity but the worst BER performance while MAP algorithm provides significantly better BER performance with highest computational complexity. 
This decoder uses MAP decoding algorithm to generate the soft output. Since the MAP decoding algorithm needs relatively large number of iterations to achieve the expected BER performance at low SNR. This leads to excessive time delay and computational complexity for deciding the system performance. The iterative nature of turbo decoding algorithms leads to increase the complexity compare to conventional FEC decoding algorithms. So for implementation of turbo code in real time system, the decoder complexity has to be reduced while preserving BER performance of the system [4].

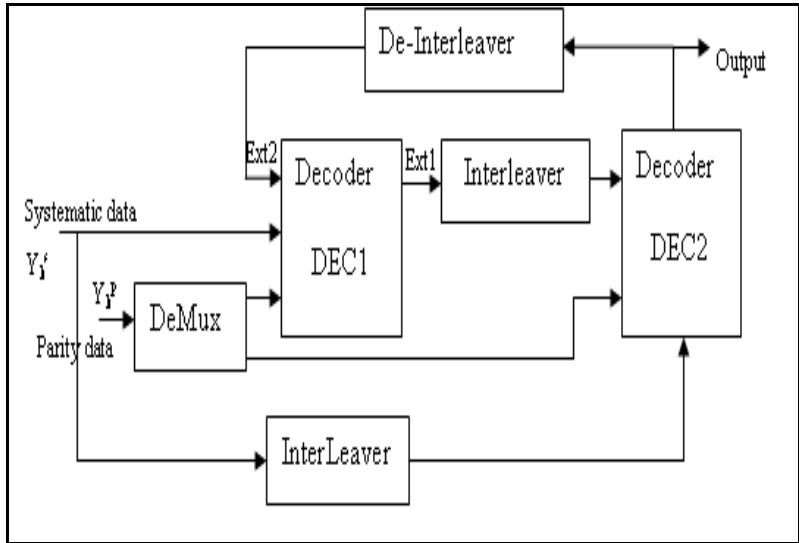

Fig 3: Turbo decoder structure for an iterative decoding process.

\subsection{Hybrid Channel Coding}

Most of the wireless applications demands uniform bit rate and good quality of service under different channel conditions. Author has suggested new scheme called Hybrid channel coding. In this scheme, we take an account of advantages of both convolution and turbo coding scheme for mobile WiMAX system. This code works like convolution codes by adapting the binary convolution encoder to convert information bit stream into the transmitted codeword. This code can be adapted when effective values of SNR fed back from channel are greater than the selected SNR threshold. The decoding is done at the receiver with reference to the same SNR threshold [5]. Thus under relatively good channel condition system adapted the convolution code and optimize the WiMAX system performance by minimizing the decoding delay and computational complexity. While on the other side, turbo code can be adapted when estimated SNRs are lower than set value of threshold SNR [8]. Turbo encoder converts a block of data into a concatenated codeword using two recursive convolution encoders and an interleaver. This technique reduces constrain length and transmitted codeword, which result in minimum power requirement at the transmitter under the poor channel conditions. In this way, hybrid channel coding helps to improve the transmission efficiency of the system. The hybrid channel coding overcomes the limitation of increase in transmitted codeword length, greater power requirement, higher decoding delay and computational complexity in Mobile WiMAX system.

\section{SIMULATION AND RESULTS}

Simulation of the hybrid channel coding scheme is carried out with reference to the parameters shown in table 1. Simulation has been tested for the generator polynomial $(7,5)$ with constrained length $\mathrm{k}=3$ of an encoder. The decoder used for convolution codes uses viterbi algorithm whereas turbo decoder uses MAP algorithm for iterations limited to two numbers has been considered for the hybrid channel coding simulation. The simulation result of a hybrid code in terms BER performance is shown in fig 4. System performance is tested by fixing the threshold SNR at $3 \mathrm{~dB}$ (for this particular simulation setup). The fixing of threshold SNR depends upon range of operation of hybrid code in terms of SNR which depends on tradeoff for BER used for wireless communication.

Table 1: Simulation parameters for hybrid channel coding.

\begin{tabular}{|l|l|}
\hline Parameters & Values \\
\hline System Bandwidth & $8.75 \mathrm{MHz}$ \\
\hline Sampling Frequency & $10 \mathrm{MHz}$ \\
\hline $\begin{array}{l}\text { Channel coding } \\
\text { methods }\end{array}$ & $\begin{array}{l}\text { Hybrid, Convolution and } \\
\text { Turbo }\end{array}$ \\
\hline Code Rates & $1 / 2$ \\
\hline Modulation schemes & $\begin{array}{l}\text { BPSK, QPSK, } \\
16 \mathrm{QAM}\end{array}$ \\
\hline Frame Size & 1024 \\
\hline Cyclic Prefix Ratio & $1 / 8$ \\
\hline Guard Interval & 128 \\
\hline SNR Range & $1-10 \mathrm{~dB}$ \\
\hline Channel & AWGN \\
\hline
\end{tabular}

The simulation has been tested for various thresholds SNR ranges from $1 \mathrm{~dB}$ to $10 \mathrm{~dB}$. The switching from one code to another occurs when fed back SNR exceeds the threshold SNR which is indicated by increasing slope from $3 \mathrm{~dB}$ to $4 \mathrm{~dB}$ in the fig 4. It is clear from figure 4 that the hybrid code is operated smoothly for expectable range of BER defined by the ITU with less power, time delay and complexity.

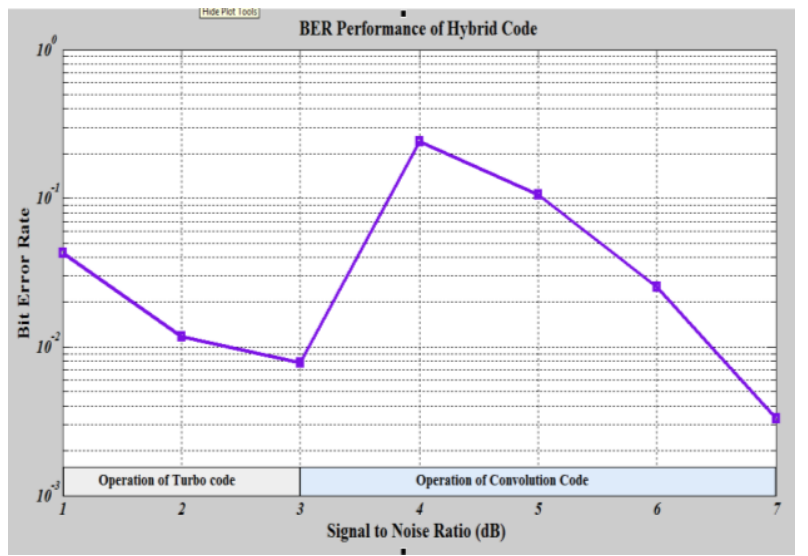

Fig 4: BER performance of Hybrid code.

After simulating the hybrid channel code by considering the above mentioned parameters, the results are compared with convolution code and the turbo code in the mobile WiMAX environment as shown in fig 5. At SNR (Estimated) lower than the threshold SNR fixed at $3 \mathrm{~dB}$, (In this case of simulation) the BER performance of hybrid code is found similar to turbo code. Indicating saving in power for transmitting the symbols compare to convolution code for the same $\operatorname{BER}\left(10^{-3}\right)$. This helps in achieving comparatively higher bit rate even under poor channel conditions. When estimated SNR detected is greater than threshold SNR of 3dB, 
the BER performance of the proposed method similar to convolution code, is degraded in percentage of error probability proportional to SNR values. Thus the decoding delay and the computational complexity are reduced by implementing the hybrid code in mobile WiMAX system. Therefore, the Mobile WiMAX system can operate with the uniform bit rate at moderate power level. The table 2 shows SNR values observed for different coding techniques and code rates with QPSK modulation.

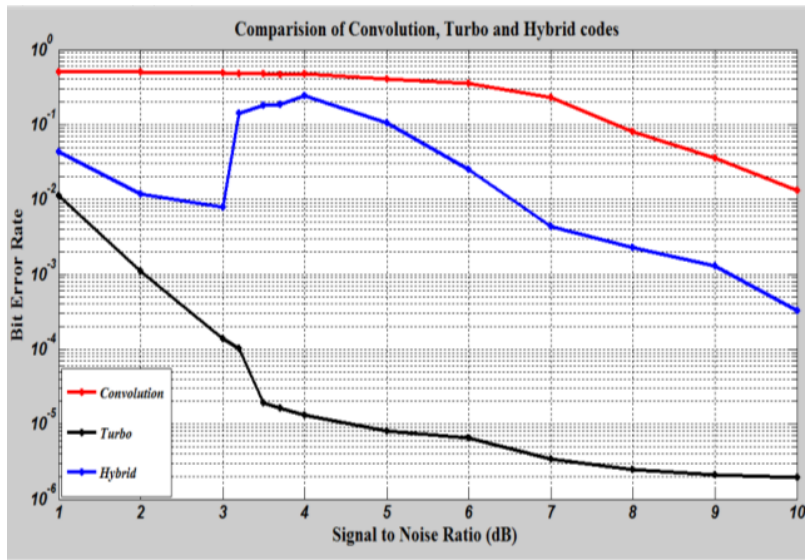

Fig 5: Comparisons of hybrid code with convolution and turbo code at threshold SNR of $3 \mathrm{~dB}$.

Similar experiments are carried out for various modulation schemes with different coding techniques to note SNR values for a particular BER. Keeping physical layer parameters same, the hybrid channel code simulation is repeated for the new threshold SNR of 5dB. The simulation results are shown in fig 6.

Table 2: SNR values for different coding techniques and code rates for QPSK modulation.

\begin{tabular}{|c|c|c|c|}
\hline $\begin{array}{c}\text { Type of } \\
\text { Codes }\end{array}$ & $\begin{array}{c}\text { Code } \\
\text { Rate } \\
\text { With } \\
\text { QPSK }\end{array}$ & $\begin{array}{r}\text { SNR at } \\
\text { BER }=10^{-2}\end{array}$ & $\begin{array}{c}\text { SNR at } \\
\text { BER }=10^{-3}\end{array}$ \\
\hline $\begin{array}{c}\text { Convolution } \\
\text { codes }\end{array}$ & $1 / 2$ & $8.1 \mathrm{~dB}$ & -- \\
\cline { 2 - 4 } & $1 / 3$ & $9.2 \mathrm{~dB}$ & -- \\
\hline $\begin{array}{c}\text { Turbo } \\
\text { Codes }\end{array}$ & $1 / 2$ & $2.1 \mathrm{~dB}$ & $2.8 \mathrm{~dB}$ \\
\cline { 2 - 4 } & $1 / 3$ & $0.5 \mathrm{~dB}$ & $1.0 \mathrm{~dB}$ \\
\hline $\begin{array}{c}\text { Hybrid } \\
\text { Code }\end{array}$ & $1 / 2$ & $2.4 \mathrm{~dB}$ & $6.6 \mathrm{~dB}$ \\
\cline { 2 - 4 } & $1 / 3$ & $3.0 \mathrm{~dB}$ & -- \\
\hline
\end{tabular}

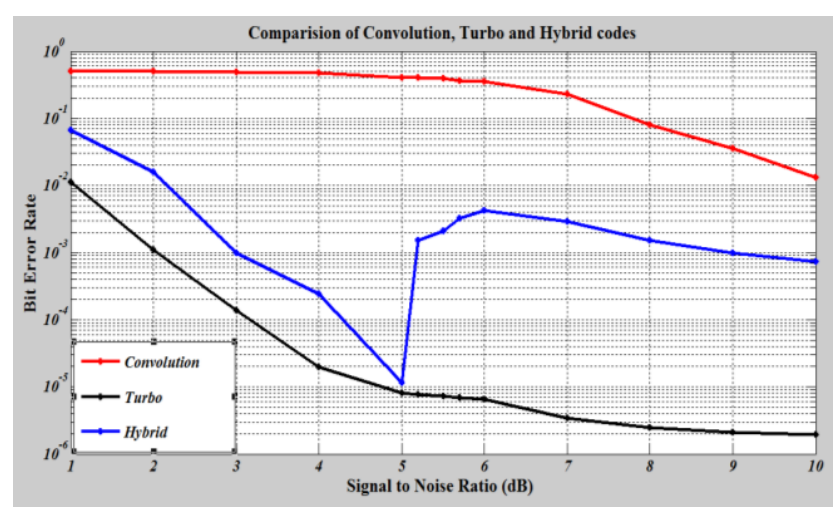

Fig 6: Comparisons of hybrid code with convolution and turbo code at threshold SNR of $5 \mathrm{~dB}$.
Test has been carried out to see the effect of various code rates on the performance of a hybrid code for WiMAX system and is shown in fig 7. It is observed that, higher code rate is more effective at a lower range of SNR and degrades slightly when SNR crosses the threshold limit.

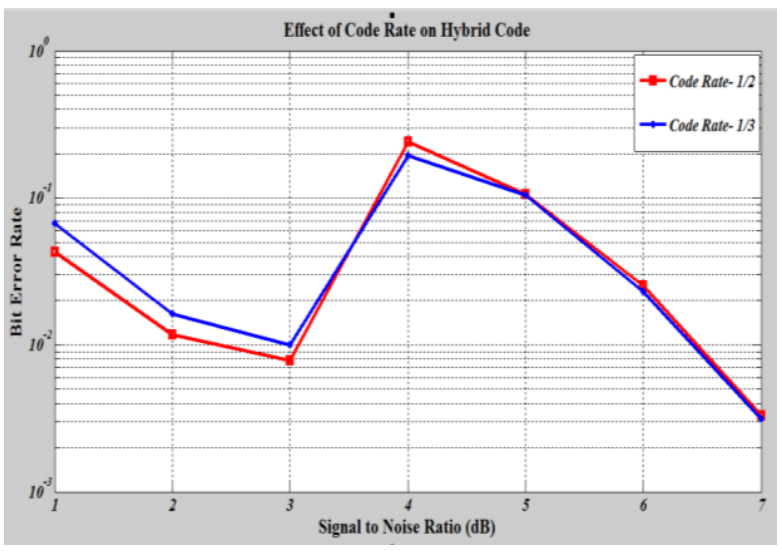

Fig 7: Effect of various code rates.

\section{CONCLUSIONS}

We have observed the BER performance of the hybrid coding scheme through the MATLAB simulation. With reference to the quality of service and channel conditions of mobile WiMAX, hybrid code performs similar to convolution code at higher SNR while its characteristics are similar to turbo codes for lower levels of SNR. Since at lower SNR system adapted turbo code principle and improved the system performance with short codeword length. Thus, the hybrid code relatively reduces the transmitted power required and helps to improve the efficiency of the Mobile WiMAX system. With the implementation of hybrid code, decoding delay of turbo decoder and computational complexity has been reduced to a certain extent. Thus, the model proposed by the author promises to provide uniform data rate which fulfils the demand of the present wireless communication system and communication link from the selection of particular coding scheme irrespective of channel conditions.

\section{ACKNOWLADGEMENT}

Authors would like to thanks Barat Sanchar Nigam Limited, Nagpur branch, for their cooperation and kind support for implementing the Hybrid channel coding technique in mobile WiMAX system.

\section{REFERENCES}

[1] Kene J. D. and Kulat K. D., 2011. Performance Evaluation of IEEE 802.16e Wi-Max Physical Layer, in Proc. of IEEE Conf. NUiCONE, 1-4.

[2] Kene J. D. and Kulat K. D., 2012. Performance Optimization of Physical Layer Using Turbo Codes: A Case Study of Wi-Max Mobile Environment, In Proc. of IEEE Conf., ET2ECN

[3] Kene J. D. and Kulat K. D., 2012. Soft Output Decoding Algorithm for Turbo Codes Implementation in Mobile Wi-Max Environment, Elsevier Vol. 6, 666-673.

[4] Kene J. D. and Kulat K. D., 2013. Iterative Decoding Termination Schemes for Turbo Code Performance Optimization In Mobile Wi-Max Environment, 
International Journal of Computer Science Issues (IJCSI), Vol. 10, Issue 4, 14-19.

[5] Kene J. D. and Kulat K. D., 2013. Channel Estimation for High Data Rate Communication in Mobile Wi-Max System, International Journal of Electronics and Communication Engineering \& Technology (IJECET), vol. 4, Issue 3, 115-123.

[6] Berrou C., 1996. Near Optimum Error Correcting Coding and Decoding: Turbo Codes, IEEE Transactions on Communications, Vol. 44, No. 1.

[7] Chen H., Sun X. and Dai L., 2010. A LDPC and Turbo Codes Hybrid Coding Scheme Based on OFDM System, in 6th International Conference on Wireless Communications Networking and Mobile computing (WiCOM), 1-4.

[8] Pauluzzi D. R. and Beaulieu N. C., 2000. A Comparison of SNR Estimation Techniques for the AWGN Cannel, IEEE Trans. Communication, Vol. 48, 1681-1691.

[9] Pyndiah R. M., 1998. Near-optimum decoding of product codes: block turbo codes, IEEE Trans. Communication, vol. 46, No. 8.

[10] Hagenauer J., Offer E. and Papke L., 1996. Iterative decoding of binary block and convolutional codes, IEEE Trans. Info. Theory, Vol. 42, No. 2.

[11] Bahl L., Cocke J., Jelinek F., and Raviv J., 1974. Optimal decoding of linear codes for minimizing symbol error rate, IEEE Trans. Inform. Theory, Vol. 20, 284287.

[12] Sadjadpour H. R. and Park F., 2000. Maximum A Posteriori decoding algorithms for turbo codes, In digital wireless communication II, Proc of SPIE, Vol. 4045, 7383.

[13] Bhise A. and Vyavahare P., 2008. Low Complexity Hybrid Turbo Codes, in Proc. of IEEE Conf. on Wireless Communications and Networking, 1050-1055.

[14] Adhicandra I., Garroppo R. G. and Giordano S., 2009. Optimizing System Capacity and Application Delays in WiMAX Networks, in Proc. of IEEE Conf. ISWCS, 540544.

[15] Wu K. and Ping L., 2004. Improved Two-state Turbo SPC code for Wireless Communication Systems, IEEE Trans. Communication, Vol.52, No.8, 1238- 1241.

[16] Wang F., Ghosh A., Sankaran C., Fleming P. J., Hsieh F., and Benes S. J., 2008. Mobile WiMAX Systems: Performance and Evolution, IEEE Communications Magazine, 41-49.

[17] S.-J. Lee, M. Goel, Y. Zhu, J.-F. Ren, and Y. Sun, Forward error correction decoding for WiMAX and
3GPP LTE modems, in Proc.42nd Asilomar Conference on Signals, Systems and Computers, 26-29 Oct. 2008, 1143-1147.

[18] IEEE 802.16-2006: IEEE Standard for Local and Metropolitan Area Networks - Part 16: Air Interface for Fixed Broadband Wireless Access Systems.

[19] IEEE. Standard 802.16-2004. Part16: Air interface for fixed broadband wireless access systems, October 2004.

[20] Andrews J. G., Ghosh A. and Wolter D. R., 2005. Broadband Wireless Access with WiMax/8O2.16: Current Performance Benchmarks and Future Potential, IEEE Communications Magazine.

Jagdish D. Kene completed his bachelor degree in Electronics Engineering in 2001, from Manoharbhai Patel Institute of Engineering and Technology, Nagpur University, Nagpur and Master degree in Electronics Engineering in 2005, from Yashavantrao Chowan College of Engineering, Nagpur University, Nagpur, M. S. India. He is currently associated with U. C. O. E. Nagpur, as Assistant Professor in Electronics Engineering Department having total experience of 11 years. He is currently pursuing $\mathrm{Ph}$. D. in the field of wireless communication under the supervision of Dr. Kishor D. Kulat at Visvervaraya National Institute of Technology (VNIT), Nagpur, M.S., India. His research work is related to Performance evaluation and optimization solution of physical layer by implementing various error correction coding techniques in mobile WiMAX environment. He has published one Journal Paper in Elsevier, three papers in International Conferences in his research area. He also published 1 paper in International Conference and more than 8 have been published in National Conferences in his academic carrier. He is member of Professional societies like ISTE. He believes that Trust and Honesty is the secrets of success.

Kishor D. Kulat completed his degree in Electrical Engineering, BE in 1980, from VRCE (at present VNIT) Nagpur and ME degree in 1984 from VJTI, Mumbai, India. He completed his Ph.D. degree in Electronics Engineering, in the year 2003 from VNIT, Nagpur. Having a total experience of more than 25 years, he is currently associated with VNIT, as Professor and Head in the Electronics \& Computer Science Department. With his profound knowledge \& experience in his field he is guiding around 24 research scholars for their doctoral degree, 11 have been awarded the Ph. D. degree. He has published around 80 Journal Papers, more than 75 papers in International Conferences \& more than 100 have been published in National Conferences. $\mathrm{He}$ has worked as Reviewer for many National \& International Conferences. He is a member of Board of Studies for Electronics Engineering, Nagpur University for last 14 years. He is member of Professional societies like IETE, IEI and ISTE. With all his faith in God, Dr. K. D. Kulat believes in achieving excellence through the process of continuous upgradation. 